\title{
Molecular Geometries at Sixth Order Møller-Plesset Perturbation Theory. At What Order Does MP Theory Give Exact Geometries?
}

\author{
Yuan He and Dieter Cremer* \\ Department of Theoretical Chemistry, Göteborg University, Reutersgatan 2, S-41320 Göteborg, Sweden
}

Received: April 18, 2000; In Final Form: May 24, 2000

\begin{abstract}
Sixth order Møller-Plesset perturbation theory (MP6) in connection with correlation consistent basis sets cc-pVDZ, cc-pVTZ, and cc-pVQZ was used to calculate accurate equilibrium geometries for 14 molecules and to establish the complete basis set (CBS) limit for MP6 with an extrapolation method that is based on CBS limit geometries obtained at second order MP (MP2) and at fourth order MP (MP4) perturbation theory. MP6 equilibrium geometries are more accurate than MP2 or MP4 geometries provided a sufficiently large basis set is used. However, improvements in the geometry are small relative to MP4 geometries where in the latter case a cancellation of correlation and basis set errors may even lead in some cases to better results than for MP6. Analysis of correlation effects reveals that MP6 will be superior to other MP methods if a bond situation is described not involving more than six electrons (single, double, or triple bonds). As soon as there is the influence of additional electron pairs as for example in the case of multiple bonds involving heteroatoms with electron lone pairs, bond lengths are slightly exaggerated due to missing disconnected eight and ten electron correlation effects. This reflects the importance of infinite order effects as provided by couple cluster methods such as CCSD or $\operatorname{CCSD}(\mathrm{T})$, which are often superior to MPn methods with $n \leq 6$.
\end{abstract}

\section{Introduction}

A basic requirement for quantum chemical methods is the prediction of correct equilibrium geometries. Normally, if a method seriously fails to reproduce the equilibrium geometry of a molecule it will also fail to provide a reliable description of the potential energy surface (PES) close to the stationary point occupied by the molecule and, because of this, of any molecular property that depends on features of the PES (energy differences, vibrational frequencies, force constants, etc.). However, one often finds that most correlation corrected ab initio methods, even though they do not provide high-accuracy equilibrium geometries, describe trends in molecular geometries reasonably well and that deviations from exact geometries are systematic. ${ }^{1-10}$

In structural chemistry, one often needs calculated molecular geometries with the highest precision possible to verify experimentally based equilibrium geometries or to use the calculated data together with spectroscopic data to derive a more precise equilibrium geometry. (See, e.g., refs 11-13.)

In the present work, accurate equilibrium geometries for 14 small molecules are calculated with many body perturbation theory (MBPT) using the Møller-Plesset (MP) perturbation operator $^{14}$ at second (MP2), fourth (MP4), and sixth order (MP6). ${ }^{2,15-17}$ This, however, is not done to present yet another set of equilibrium geometries for molecules, for which rather exact experimental geometries are already available. On the contrary, we want to use experimental geometries to determine errors in calculated geometries at various levels of MP theory and to identify with the help of these errors typical electron correlation effects needed to correctly describe molecular properties such as geometries. For this purpose, we have to separate basis set and correlation errors, which can only be done by extrapolating at a given level of theory to the complete basis

* E-mail: Cremer@theoc.gu.se. set (CBS) limit and, then, to compare CBS values with experiment.

CBS limit geometries at the MP6 level of theory are determined in this work using a new extrapolation method based on previous work by Dunning and others. ${ }^{18,19}$ The extrapolation method implies that first the dependence of the MP2 or MP4 value of a given geometrical parameter on the basis set size is determined to calculate the corresponding MP2 and MP4 CBS limit values. The relationship between calculated value and basis set size found at the MP2 and MP4 level of theory is then used to predict the MP6 CBS limit geometry under the assumption that the basis set dependence is similar at different levels of MP $n$ theory. Actually, the same assumption is the basis of the Gp $(p=1,2,3)$ methods of Curtiss, Raghavachari, and Pople ${ }^{20}$ who make even the additional assumption that basis set dependencies calculated at the MP2 and MP4 level of theory can also be assumed to be valid at the coupled cluster (CC) level of theory, namely at the QCISD(T) level. However, we will discuss in the following that such an assumption has to be used with care because the basis set dependence of $\mathrm{MPn}$ properties can differ from that of $\mathrm{CC}(\mathrm{QCI})$ properties.

The MP6 geometries calculated in this work will be compared with reliable experimental geometries with the aim of clarifying which electron correlation effects are needed to determine highly accurate geometries. This in turn will provide a basis to predict the degree of accuracy achieved for geometries calculated at a given level of MPn theory. We will discuss in this connection the basic question whether MPn theory at order $n \leq 6$ is a useful quantum chemical method because this has been questioned in recent times. ${ }^{21}$ Also we will comment on the performance of $\mathrm{CC}$ methods in comparison to that of MPn methods because MP and CC theory are closely related. Finally, guidance will be given how to obtain reliable equilibrium geometries from quantum chemical calculations. 


\section{Determination of MP6 Geometries with a Complete Basis Set}

The results presented in this work are based on the use of (a) MP6 theory, (b) correlation consistent basis sets, (c) suitable extrapolation formulas, (d) a detailed analysis of electron correlation effects, and (e) an error analysis of calculated geometries. These five ingredients will be described in the following.

MP6 Theory. Two levels of MP6 theory were applied in this work, namely complete MP6 and MP6(M7), the computational cost of which scale with $\mathrm{O}\left(\mathrm{M}^{9}\right)$ and $\mathrm{O}\left(\mathrm{M}^{7}\right)(\mathrm{M}$ : number of basis functions). ${ }^{15}$ MP6(M7) is obtained by dropping all $\mathrm{O}\left(\mathrm{M}^{9}\right)$ and $\mathrm{O}\left(\mathrm{M}^{8}\right)$ terms of the MP6 correlation energy. This is justified in view of previous investigations, which revealed that these terms represent a relative small fraction (ca. 12\%) of the total MP6 correlation energy because of the cancellation of positive and negative terms. ${ }^{15,16}$ Hence, MP6(M7) represents a reasonable and economically attractive alternative to the costly MP6 method.

All MP6 calculations (full core) were carried out with the program of $\mathrm{He}$ and Cremer, ${ }^{15}$ which is part of the program package COLOGNE99. ${ }^{23}$ Since analytical energy gradients are not available at this level of theory, geometry optimization was performed numerically using the Davidon-Fletcher-Powell algorithm. ${ }^{24}$ For the 14 molecules considered, bond lengths and bond angles were calculated with an accuracy of $10^{-4} \AA$ and $10^{-2}$ degrees. Generally, however, experimental errors are one magnitude larger so that it is meaningful to discuss changes of $0.001 \AA$ and 0.1 degree. On the other hand, calculated geometries may differ marginally so that differences will only become obvious if bond lengths and angles are reported with higher accuracy. This required more stringent convergence criteria in both the SCF and the geometry optimizations than are normally applied. Therefore, a convergence criterion of $10^{-8}$ with regard to changes in the density matrix was used for the SCF iterations and $10^{-5}$ with regard to changes in the forces (expressed in mdyn/ $/$ ) for the geometry optimization.

Basis Sets. All calculations carried out in this work were performed with Dunning's correlation consistent polarized basis sets cc-pVmZ where the cardinal number $m$ was chosen to be 2, 3, and $4 .^{25}$ The corresponding basis sets are of VDZ+P, $\mathrm{VTZ}+\mathrm{P}$, and VQZ+P quality given by $(9 \mathrm{~s} 4 \mathrm{p} 1 \mathrm{~d} / 4 \mathrm{~s} 1 \mathrm{p})[3 \mathrm{~s} 2 \mathrm{p} 1 \mathrm{~d} /$ $2 \mathrm{~s} 1 \mathrm{p}$ ], (10s5p2d1f/5s2p1d) [4s3p2d1f/3s2p1d], and (12s6p3d2f1g/ $6 \mathrm{~s} 3 \mathrm{p} 2 \mathrm{~d} 1 \mathrm{f})[5 \mathrm{~s} 4 \mathrm{p} 3 \mathrm{~d} 2 \mathrm{f} 1 \mathrm{~g} / 4 \mathrm{~s} 3 \mathrm{p} 2 \mathrm{~d} 1 \mathrm{f}]$ contractions, respectively. ${ }^{25}$ Dunning and co-workers ${ }^{26-28}$ showed that these basis sets provide an excellent platform to investigate calculated molecular properties with regard to their dependence on the basis set chosen. Since cc-pVmZ results obtained with correlation corrected ab initio methods monotonically converge to a limit value, one can use them to extrapolate to the CBS limit value of a given molecular property (energy, bond length, dipole moment, etc.).

Calculation of CBS Limits at MP2 and MP4. A number of different extrapolation procedures are suggested in the literature, ${ }^{18,19}$ which are all based on an exponential convergence behavior of the value of the property in question, i.e., changes in the property value decrease exponentially and become zero for the CBS limit reached with an infinitely large basis set. Several extrapolation equations suggested by Dunning and coworkers ${ }^{18}$ were tested in this work and eq 1 turned out to best suited (in terms of applicability, accuracy, and interpretability) for extrapolating to the CBS limit:

$$
Q(m)=Q(\infty)+a \mathrm{e}^{-\mathrm{m}}+b \mathrm{e}^{-\mathrm{m}^{2}}
$$

According to eq 1 , the geometrical parameter $Q$ (bond length, bond angle) is expressed as a function of the cardinal number $\mathrm{m}$ of the basis sets used, $Q(\infty)$ is the estimated CBS limit of property $Q$, and $a, b$ are the fitting parameters, which (together with $Q(\infty)$ ) can be determined in a least-squares minimization procedure. ${ }^{18}$ Equation 1 provides a simple basis for discussing the convergence behavior of the $Q(m)$ series because the term $a \mathrm{e}^{-\mathrm{m}}$ represents a slower and the term $b \mathrm{e}^{-\mathrm{m}^{2}}$ a faster converging series of $Q(m)$ values. Hence, $a>b$ characterizes a geometrical parameter $Q$ as (relatively) slowly converging and, therefore, difficult to calculate while $a<b$ indicates faster convergence and a chance of obtaining a reliable value for $Q$ already with a basis set of modest size.

Contrary to previous work by Dunning and co-workers ${ }^{18,26}$ who used four or five points to determine the convergence behavior of $Q(m)$ in a least-squares sense, in this work, a threepoint extrapolation procedure was used for all molecules considered, which sacrifices some of the accuracy of calculated CBS values, but is less costly and provides a simple basis to compare the convergence properties of different geometry parameters at different levels of MPn theory.

Error Analysis. Deviations of calculated geometrical parameters from the corresponding experimental values were analyzed using mean absolute deviation $\mu_{\mathrm{abs}}$, mean deviation $\mu$, and standard deviation $\sigma$ :

$$
\begin{gathered}
\mu_{\mathrm{abs}}=\frac{1}{n} \sum_{i=1}^{n}\left|\Delta_{i}\right|, \text { for } \Delta_{i}=R_{i}(\mathrm{cal})-R_{i}(\exp ) \\
\mu=\frac{1}{n} \sum_{i=1}^{n} \Delta_{i} \\
\sigma=\sqrt{\frac{\sum_{i=1}^{n}\left(\Delta_{i}-\mu\right)^{2}}{n}}
\end{gathered}
$$

where $R_{i}(\mathrm{cal})$ denotes a calculated and $R_{i}(\exp )$ the corresponding experimental value of a geometrical parameter $R=\{r, \theta, \tau\}$. Utilizing mean deviation $\mu$ and standard deviation $\sigma$, the normal distribution curve was calculated according to eq 5 .

$$
\rho(\Delta)=\frac{1}{\sqrt{2 \pi \sigma}} \exp \left[\frac{-(\Delta-\mu)^{2}}{2 \sigma^{2}}\right]
$$

Calculation of CBS Limits at MP6. It is well-known that MP $n$ energies as well as other molecular properties calculated at $\mathrm{MP} n$ do not converge monotonically to a limit value with increasing order $n$ of perturbation theory, but oscillate where oscillations seem to become smaller for increasing order $n .^{2,3,30}$ This convergence behavior was found to be a result of the stepwise introduction of higher electron correlation effects into MPn theory. ${ }^{2,16}$ At even orders $n$, new correlation effects are included while at odd orders corrections to these new correlation effects are added.

$M P 2$. Pair correlation effects described by the double (D) excitations; since correlation effects are not coupled, pair correlation is exaggerated.

Third-Order MP (MP3). Corrections to pair correlations caused by a coupling of D excitations.

MP4. Three-electron correlation effects described by triple (T) excitations; orbital relaxation effects are described by single 
TABLE 1: Comparison of Extrapolated MP4 Geometries with Calculated Values ${ }^{a}$

\begin{tabular}{|c|c|c|c|c|c|c|c|c|c|}
\hline \multirow[b]{2}{*}{ molecule } & \multirow{2}{*}{$\begin{array}{l}\text { geom. } \\
\text { parameter }\end{array}$} & \multicolumn{2}{|c|}{ MP2 fitting coefficient } & \multicolumn{3}{|c|}{ extrapolated geometries $R(\text { extrpl })^{b}$} & \multicolumn{3}{|c|}{$R($ extrpl $)-R(\text { calculated })^{c}$} \\
\hline & & $a$ & $b$ & cc-pVTZ & cc-pVQZ & cc-pVœZ & cc-pVTZ & cc-pVQZ & $c c-p V \infty Z$ \\
\hline $\mathrm{CH}_{2}\left({ }^{3} B_{1}\right)$ & $\begin{array}{l}r(\mathrm{CH}) \\
\theta(\mathrm{HCH})\end{array}$ & $\begin{array}{r}-0.0227 \\
2.9709\end{array}$ & $\begin{array}{r}1.2256 \\
-91.4686\end{array}$ & $\begin{array}{r}1.0730 \\
133.38\end{array}$ & $\begin{array}{l}1.0736 \\
133.30\end{array}$ & $\begin{array}{c}1.0740 \\
133.25\end{array}$ & $\begin{array}{l}0.0012 \\
-0.30\end{array}$ & $\begin{array}{l}0.0005 \\
-0.20\end{array}$ & $\begin{array}{l}-0.0001 \\
-0.13\end{array}$ \\
\hline $\mathrm{CH}_{4}\left({ }^{1} A_{1}\right)$ & $r(\mathrm{CH})$ & 0.0219 & 0.7825 & 1.0859 & 1.0851 & 1.0847 & 0.0003 & 0.0000 & -0.0002 \\
\hline $\mathrm{HCN}\left({ }^{1} \Sigma\right)$ & $\begin{array}{l}r(\mathrm{CH}) \\
r(\mathrm{CH})\end{array}$ & $\begin{array}{r}0.0870 \\
-0.0890\end{array}$ & $\begin{array}{l}0.5799 \\
1.4513\end{array}$ & $\begin{array}{l}1.1630 \\
1.0628\end{array}$ & $\begin{array}{l}1.1602 \\
1.0655\end{array}$ & $\begin{array}{l}1.1586 \\
1.0671\end{array}$ & $\begin{array}{r}-0.0013 \\
0.0012\end{array}$ & $\begin{array}{r}-0.0014 \\
0.0007\end{array}$ & $\begin{array}{r}-0.0015 \\
0.0005\end{array}$ \\
\hline $\mathrm{HCN}\left({ }^{1} \sum\right)$ & $\begin{array}{l}r(\mathrm{HN}) \\
r(\mathrm{NC})\end{array}$ & $\begin{array}{r}-0.0328 \\
0.0460\end{array}$ & $\begin{array}{l}0.8421 \\
0.8462\end{array}$ & $\begin{array}{l}0.9939 \\
1.1782\end{array}$ & $\begin{array}{l}0.9948 \\
1.1766\end{array}$ & $\begin{array}{l}0.9954 \\
1.1758\end{array}$ & $\begin{array}{l}0.0003 \\
0.0007\end{array}$ & $\begin{array}{l}0.0002 \\
0.0008\end{array}$ & $\begin{array}{l}0.0002 \\
0.0009\end{array}$ \\
\hline $\mathrm{CO}\left({ }^{1} \Sigma^{+}\right)$ & $r(\mathrm{CO})$ & 0.1015 & 0.1495 & 1.1431 & 1.1399 & 1.1380 & 0.0006 & 0.0009 & 0.0010 \\
\hline $\mathrm{NH}_{3}\left({ }^{1} A_{1}\right)$ & $\begin{array}{l}r(\mathrm{NH}) \\
\theta(\mathrm{HNH})\end{array}$ & $\begin{array}{r}0.0328 \\
-8.9542\end{array}$ & $\begin{array}{r}0.6054 \\
-88.3837\end{array}$ & $\begin{array}{l}1.0120 \\
105.98\end{array}$ & $\begin{array}{l}1.0109 \\
106.28\end{array}$ & $\begin{array}{l}1.0103 \\
106.44\end{array}$ & $\begin{aligned} & 0.0005 \\
- & 0.04\end{aligned}$ & $\begin{array}{l}0.0003 \\
-0.02\end{array}$ & $\begin{aligned} & 0.0002 \\
- & 0.02\end{aligned}$ \\
\hline $\mathrm{N}_{2}\left({ }^{1} \Sigma_{g}^{+}\right)$ & $r(\mathrm{NN})$ & 0.0749 & 0.7000 & 1.1074 & 1.1050 & 1.1036 & -0.0025 & -0.0029 & -0.0032 \\
\hline $\mathrm{N}_{2} \mathrm{H}_{2}\left({ }^{1} A_{g}\right)$ & $\begin{array}{l}r(\mathrm{NH}) \\
r(\mathrm{NN}) \\
\theta(\mathrm{NNH})\end{array}$ & $\begin{array}{r}0.0265 \\
0.0695 \\
-8.6963\end{array}$ & $\begin{array}{r}0.6683 \\
0.4827 \\
-9.3744\end{array}$ & $\begin{array}{l}1.0292 \\
1.2526 \\
105.70\end{array}$ & $\begin{array}{r}1.0283 \\
1.2504 \\
105.97\end{array}$ & $\begin{array}{r}1.0278 \\
1.2491 \\
106.13\end{array}$ & $\begin{array}{l}0.0007 \\
-0.0021 \\
0.09\end{array}$ & $\begin{array}{l}0.0004 \\
-0.0025 \\
0.09\end{array}$ & $\begin{aligned} 0.0002 \\
-0.0027 \\
0.09\end{aligned}$ \\
\hline $\mathrm{HNO}\left({ }^{1} A^{\prime}\right)$ & $\begin{array}{l}r(\mathrm{NO}) \\
r(\mathrm{HN}) \\
\theta(\mathrm{HNO})\end{array}$ & $\begin{array}{r}0.0672 \\
0.0283 \\
-2.7056\end{array}$ & $\begin{array}{r}0.1710 \\
0.7696 \\
-3.8687\end{array}$ & $\begin{array}{r}1.2172 \\
1.0553 \\
107.85\end{array}$ & $\begin{array}{r}1.2150 \\
1.0543 \\
107.94\end{array}$ & $\begin{array}{r}1.2138 \\
1.0538 \\
107.99\end{array}$ & $\begin{array}{c}-0.0029 \\
0.0015 \\
0.09\end{array}$ & $\begin{array}{c}-0.0038 \\
0.0015 \\
0.12\end{array}$ & $\begin{array}{c}-0.0042 \\
0.0016 \\
0.14\end{array}$ \\
\hline $\mathrm{H}_{2} \mathrm{O}\left({ }^{1} A_{1}\right)$ & $\begin{array}{l}r(\mathrm{OH}) \\
\theta(\mathrm{HOH})\end{array}$ & $\begin{array}{r}0.0472 \\
-16.0343\end{array}$ & $\begin{array}{r}0.1500 \\
-17.5339\end{array}$ & $\begin{array}{c}0.9587 \\
103.59\end{array}$ & $\begin{array}{l}0.9572 \\
104.10\end{array}$ & $\begin{array}{c}0.9563 \\
104.39\end{array}$ & $\begin{array}{l}0.0002 \\
-0.01\end{array}$ & $\begin{array}{l}0.0000 \\
-0.01\end{array}$ & $\begin{array}{l}-0.0002 \\
-0.01\end{array}$ \\
\hline $\mathrm{H}_{2} \mathrm{O}_{2}\left({ }^{1} A\right)$ & $\begin{array}{l}r(\mathrm{OO}) \\
r(\mathrm{OH}) \\
\theta(\mathrm{OOH}) \\
\tau(\mathrm{HOOH})\end{array}$ & $\begin{array}{r}0.1027 \\
0.0472 \\
-9.6459 \\
39.0790\end{array}$ & $\begin{array}{r}0.0334 \\
0.1611 \\
9.7362 \\
88.2633\end{array}$ & $\begin{array}{c}1.4598 \\
0.9638 \\
99.33 \\
113.15\end{array}$ & $\begin{array}{c}1.4566 \\
0.9623 \\
99.64 \\
111.90\end{array}$ & $\begin{array}{c}1.4547 \\
0.9614 \\
99.81 \\
111.19\end{array}$ & $\begin{array}{l}0.0011 \\
0.0002 \\
0.08 \\
-0.98\end{array}$ & $\begin{array}{c}0.0002 \\
-0.0001 \\
0.07 \\
-0.99\end{array}$ & $\begin{array}{c}-0.0004 \\
-0.0004 \\
0.07 \\
-0.99\end{array}$ \\
\hline $\mathrm{FH}\left({ }^{1} \Sigma^{+}\right)$ & $r(\mathrm{FH})$ & 0.0270 & -0.0041 & 0.9171 & 0.9163 & 0.9158 & 0.0000 & -0.0002 & -0.0003 \\
\hline $\mathrm{F}_{2}\left({ }^{1} \Sigma_{g}^{+}\right)$ & $r(\mathrm{FF})$ & 0.0106 & 1.4932 & 1.4223 & 1.4218 & 1.4216 & 0.0072 & 0.0069 & 0.0067 \\
\hline $\operatorname{HOF}\left({ }^{1} A^{\prime}\right)$ & $\begin{array}{l}r(\mathrm{OH}) \\
r(\mathrm{OF}) \\
\theta(\mathrm{HOF})\end{array}$ & $\begin{array}{r}0.0382 \\
0.0124 \\
-6.3545\end{array}$ & $\begin{array}{r}0.2258 \\
0.8990 \\
-3.2726\end{array}$ & $\begin{array}{l}0.9672 \\
1.4419 \\
97.16\end{array}$ & $\begin{array}{l}0.9660 \\
1.4414 \\
97.36\end{array}$ & $\begin{array}{l}0.9653 \\
1.4412 \\
97.47\end{array}$ & $\begin{array}{l}0.0000 \\
0.0023 \\
-0.05\end{array}$ & $\begin{aligned}-0.0004 \\
0.0014 \\
0.01\end{aligned}$ & $\begin{array}{c}-0.0005 \\
0.0008 \\
0.04\end{array}$ \\
\hline $\begin{array}{l}\text { mean absolut } \\
\text { bond lengt } \\
\text { bond angle }\end{array}$ & ation & & & & & & $\begin{array}{l}0.0013 \\
0.09\end{array}$ & $\begin{array}{l}0.0013 \\
0.08\end{array}$ & $\begin{array}{l}0.0013 \\
0.07\end{array}$ \\
\hline
\end{tabular}

${ }^{a}$ Bond lengths are given in $\AA$ and angles in degrees. $R$ denotes the geometrical parameters $r, \theta$, and $\tau$. ${ }^{b}$ Extrapolated values are obtained from eq 1 utilizing coefficients $a$, and $b$ determined at the MP2 level. ${ }^{c}$ The calculated geometries $R$ (calculated) are listed in Table 3.

(S) excitations; pair-pair correlation effects are described by disconnected quadruple (Q) excitations.

Fifth Order MP (MP5). Couplings between three-electron correlation, orbital relaxation, and pair-pair correlation effects.

MP6. Four-electron correlation effects are described by connected Q excitations, pair-pair-pair correlation effects are described by hextuple $(\mathrm{H})$ excitations, combinations of 2- and 3 -electron correlation effects are described by pentuple (P) excitations.

The trends in calculated MPn correlation energies affect all energy-dependent molecular properties that show a similar oscillatory behavior.

For an oscillating MPn series it is difficult to extrapolate calculated MP2, MP3, MP4, etc. values to the MP $\infty$ value, which should be identical to the full CI (FCI) value of the property in question. However, the analysis of MPn results shows that in the case of initial oscillations there are often two converging series, namely one for even order results and one for odd order results, which converge to the same limit. ${ }^{15-17}$ Hence, when considering MP2, MP4, and MP6 results one has a reasonable basis to predict results which one would obtain at higher levels of MPn theory. There might be, of course, the danger that oscillations increase for increasing $n$ thus leading to a divergent series as was discussed in recent FCI work of Olsson and co-workers. ${ }^{21}$ Arguments have been presented that divergence of the MPn series is a result of either strong multireference character of the system considered or the use of an unbalanced basis set. ${ }^{30}$ In the present work, closed-shell molecules without any multireference character are investigated with well-balanced basis sets so that the problem of a diverging $\mathrm{MP} n$ series either for energies or other molecular properties does not exist.

For geometrical parameters such as bond lengths and bond angles, the convergence behavior of the $Q(m)$ series calculated at the MP2, MP4, and MP6 level of theory is similar as was confirmed by appropriate MPn calculations. First, coefficients $a, b$, and the CBS limit $\mathrm{Q}(\infty)$ of the geometrical parameter $Q$ were determined at the MP2 level by a three-point extrapolation procedure employing the cc-pVDZ, cc-pVTZ, and cc-pVQZ basis sets. Then, the MP2 fitting coefficients were used in connection with a MP4/cc-pVDZ calculation to predict values of geometrical parameters at the MP4/cc-pVTZ, MP4/cc-pVQZ, and MP4/CBS levels of theory (Table 1). Finally, predicted values were compared with directly calculated MP4/cc-pVTZ, MP4/cc-pVQZ, and MP4/CBS values of $Q$.

As shown by Table 1 , the mean absolute deviation $|\mu|$ for 20 bond lengths and seven angles is about $0.001 \AA$ and 0.1 degree, respectively. The normal distribution of errors of predicted and calculated MP4/CBS limit values with regard to experimental geometrical parameters ${ }^{31-40}$ are almost identical (see Figure 1), i.e., MP4/CBS limit values determined at the MP4 level with three points or with just one point using the curve $Q(m)$ determined at the MP2 level differ only slightly. 


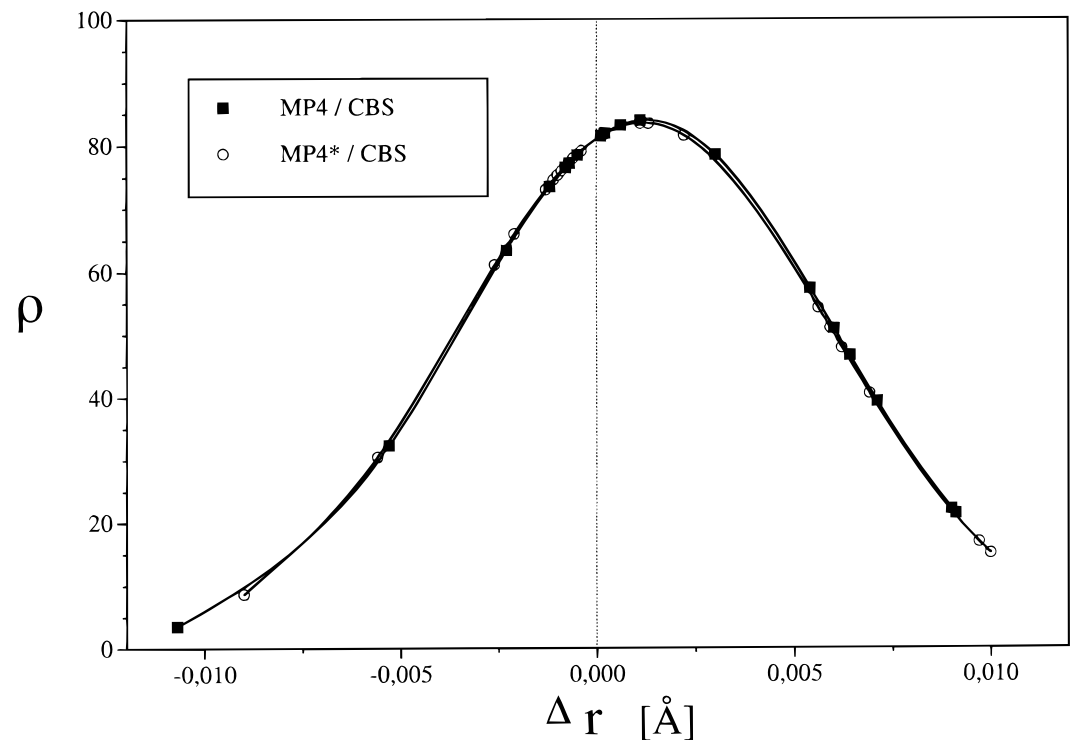

Figure 1. Normal distribution of errors for calculated MP4/CBS bond lengths. Filled circles denote values obtained from MP4/cc-pVmZ calculations $(m=2,3,4)$ and eq 1 , open circles values obtained from MP4/cc-VDZ calculations combined with MP2 fitting coefficients $a$ and $b$ (indicated by $\mathrm{MP} 4 * / \mathrm{CBS})$.

TABLE 2: Comparison of Calculated and Estimated MP6/cc-pVTZ Geometries ${ }^{a}$

\begin{tabular}{|c|c|c|c|c|c|c|c|}
\hline \multirow[b]{2}{*}{ molecule } & \multirow{2}{*}{$\begin{array}{c}\text { geom. } \\
\text { parameter }\end{array}$} & \multirow{2}{*}{$\begin{array}{c}\text { MP6 } \\
\text { extrapolated }\end{array}$} & \multicolumn{2}{|c|}{ MP6(M7) } & \multirow[b]{2}{*}{$\Delta 7^{b}$} & \multicolumn{2}{|c|}{ MP6 } \\
\hline & & & calculated & $\Delta$ & & $\overline{\mathrm{MP} 6(\mathrm{M} 7)+\Delta 7^{c}}$ & $\Delta$ \\
\hline $\mathrm{CH}_{2}\left({ }^{3} B_{1}\right)$ & $\begin{array}{l}r(\mathrm{CH}) \\
\theta(\mathrm{HCH})\end{array}$ & $\begin{array}{l}1.0735 \\
133.90\end{array}$ & $\begin{array}{l}1.0784 \\
133.51\end{array}$ & $\begin{array}{c}-0.0049 \\
0.39\end{array}$ & $\begin{array}{l}0.0001 \\
0.00\end{array}$ & $\begin{array}{l}1.0785 \\
133.51\end{array}$ & $\begin{array}{l}-0.0050 \\
0.39\end{array}$ \\
\hline $\mathrm{HCN}\left({ }^{1} \Sigma\right)$ & $\begin{array}{l}r(\mathrm{CN}) \\
r(\mathrm{CH})\end{array}$ & $\begin{array}{l}1.1599 \\
1.0623\end{array}$ & $\begin{array}{l}1.1636 \\
1.0669\end{array}$ & $\begin{array}{l}-0.0037 \\
-0.0046\end{array}$ & $\begin{array}{l}0.0008 \\
0.0000\end{array}$ & $\begin{array}{l}1.1644 \\
1.0669\end{array}$ & $\begin{array}{l}-0.0045 \\
-0.0046\end{array}$ \\
\hline $\mathrm{HNC}\left({ }^{1} \Sigma\right)$ & $\begin{array}{l}r(\mathrm{HN}) \\
r(\mathrm{NC})\end{array}$ & $\begin{array}{l}0.9939 \\
1.1773\end{array}$ & $\begin{array}{l}0.9962 \\
1.1796\end{array}$ & $\begin{array}{l}-0.0023 \\
-0.0023\end{array}$ & $\begin{array}{l}0.0002 \\
0.0014\end{array}$ & $\begin{array}{l}0.9964 \\
1.1810\end{array}$ & $\begin{array}{l}-0.0025 \\
-0.0037\end{array}$ \\
\hline $\mathrm{CO}\left({ }^{1} \Sigma^{+}\right)$ & $r(\mathrm{CO})$ & 1.1432 & 1.1473 & -0.0041 & 0.0029 & 1.1502 & -0.0070 \\
\hline $\mathrm{NH}_{3}\left({ }^{1} A_{1}\right)$ & $\begin{array}{l}r(\mathrm{NH}) \\
\theta(\mathrm{HNH})\end{array}$ & $\begin{array}{l}1.0124 \\
105.96\end{array}$ & $\begin{array}{l}1.0144 \\
105.67\end{array}$ & $\begin{array}{c}-0.0020 \\
0.29\end{array}$ & $\begin{array}{l}0.0002 \\
-0.02\end{array}$ & $\begin{array}{l}1.0146 \\
105.65\end{array}$ & $\begin{array}{l}-0.0022 \\
0.32\end{array}$ \\
\hline $\mathrm{N}_{2}\left({ }^{1} \sum_{g}^{+}\right)$ & $r(\mathrm{NN})$ & 1.1049 & 1.1080 & -0.0031 & 0.0009 & 1.1089 & -0.0040 \\
\hline $\mathrm{H}_{2} \mathrm{O}\left({ }^{1} A_{1}\right)$ & $\begin{array}{l}r(\mathrm{OH}) \\
\theta(\mathrm{HOH})\end{array}$ & $\begin{array}{l}0.9587 \\
103.56\end{array}$ & $\begin{array}{l}0.9597 \\
103.52\end{array}$ & $\begin{array}{c}-0.0010 \\
0.04\end{array}$ & $\begin{array}{l}0.0002 \\
-0.02\end{array}$ & $\begin{array}{l}0.9599 \\
103.50\end{array}$ & $\begin{array}{l}-0.0012 \\
0.06\end{array}$ \\
\hline $\mathrm{FH}\left({ }^{1} \Sigma^{+}\right)$ & $r(\mathrm{FH})$ & 0.9179 & 0.9177 & 0.0002 & 0.0001 & 0.9178 & 0.0001 \\
\hline $\mathrm{F}_{2}\left({ }^{1} \Sigma_{g}^{+}\right)$ & $r(\mathrm{FF})$ & 1.4243 & 1.4155 & 0.0088 & 0.0032 & 1.4187 & 0.0056 \\
\hline $\begin{array}{l}\text { mean absolut } \\
\text { bond lengt } \\
\text { bond angle }\end{array}$ & & & & $\begin{array}{l}0.0034 \\
0.24\end{array}$ & & & $\begin{array}{l}0.0037 \\
0.26\end{array}$ \\
\hline
\end{tabular}

${ }^{a}$ Bond lengths are given in $\AA$ and angles in degrees. Extrapolated values are based on MP6(M7)/cc-pVDZ calculations and fitting coefficients determined at the MP4 level in connection with eq $1 . \Delta$ gives the difference between extrapolated and calculated geometrical parameters. ${ }^{b} \Delta 7$ denotes the difference $R(\mathrm{MP} 6)-R\left(\mathrm{MP} 6(\mathrm{M} 7)\right.$ ) determined at the MP6/cc-pVDZ level of theory. ${ }^{c}$ MP6 geometries are obtained by adding $\Delta 7$ to $R(\mathrm{MP6}(\mathrm{M} 7))$.

A second test was made at the MP6 level using the extrapolation function $Q(m)$ determined at the MP4 level. At the MP6(M7)/cc-pVTZ level of theory, calculated and predicted bond lengths (angles) differ by just $0.003 \AA$ ( 0.2 degree; mean absolute deviation). If correction increments for the difference $Q($ MP6) $-Q($ MP6(M7)) obtained with the cc-pVDZ basis are added, mean absolute deviations are $0.004 \AA$ and 0.3 degree, respectively (Table 2).

The results of the two tests summarized in Tables 1 and 2 indicate that the fitting coefficients $a$ and $b$ are comparable at MP2, MP4, and MP6, which is not the case when mixing results from even and odd orders of MPn theory. Although MP6 geometries could be calculated with the cc-pVTZ basis set only in selected cases because of the $O\left(M^{9}\right)$ dependence on the number $M$ of basis functions, it is reasonable to extrapolate MP6/ cc-pVDZ results with the help of the calculated MP4 fitting coefficients $a$ and $b$ to MP6/CBS limit geometries. MP6 geometries obtained in this way are listed in Table 3 together with directly calculated MP6 values. For reasons of comparison, the corresponding MP2 and MP4 geometries as well as the corresponding experimental values ${ }^{31-40}$ are also shown in Table 3.

\section{Comparison of MP Geometries with Experimental Geometries}

Calculated and experimental geometries are compared in Figures 2 and 3 by displaying the normal distribution of the errors in calculated bond lengths (20 values) obtained at a given level of theory. The position of the maximum of the distribution curve corresponds to the mean deviation $\mu$ while the half-width of the curve reflecting the scattering of errors is defined by the standard deviation $\sigma$. These parameters are also given together 
TABLE 3: Comparison Experimental Geometries with MPn Geometries Calculated with cc-pVmZ Basis Sets ${ }^{a}$

\begin{tabular}{|c|c|c|c|c|c|c|c|c|c|c|c|c|c|c|c|}
\hline \multirow[b]{2}{*}{ molecule } & \multirow{2}{*}{$\begin{array}{c}\text { geom. } \\
\text { parameter }\end{array}$} & \multicolumn{4}{|c|}{ MP2 } & \multicolumn{4}{|c|}{ MP4 } & \multicolumn{4}{|c|}{ MP6 } & \multirow[b]{2}{*}{ expt } & \multirow[b]{2}{*}{ ref } \\
\hline & & cc-pVDZ & cc-pVTZ & cc-pVQZ & cc-pVœZ & cc-pVDZ & cc-pVTZ & cc-pVQZ & cc-pVœZ & cc-pVDZ & cc-pVTZ & cc-pVQZ & $\mathrm{cc}-\mathrm{pV} \propto \mathrm{Z}$ & & \\
\hline \multirow[t]{2}{*}{$\mathrm{CH}_{2}\left({ }^{3} B_{1}\right)$} & $r(\mathrm{CH})$ & 1.0893 & 1.0689 & 1.0695 & 1.0699 & 1.0934 & 1.0718 & 1.0732 & 1.0741 & 1.0951 & 1.0785 & 1.0749 & 1.0758 & 1.0753 & \multirow{2}{*}{$33 \mathrm{a}, r_{e}$} \\
\hline & $\theta(\mathrm{HCH})$ & 131.77 & 133.18 & 133.10 & 133.04 & 131.97 & 133.69 & 133.50 & 133.38 & 132.18 & 133.51 & 133.71 & 133.59 & 133.93 & \\
\hline $\mathrm{CH}_{4}\left({ }^{1} A_{1}\right)$ & $r(\mathrm{CH})$ & 1.0985 & 1.0824 & 1.0816 & 1.0812 & 1.1020 & 1.0857 & 1.0852 & 1.0850 & 1.1026 & 1.0863 & 1.0858 & 1.0856 & 1.0857 & $33 \mathrm{~b}, r_{e}$ \\
\hline \multirow[t]{2}{*}{$\mathrm{HCN}\left({ }^{1} \Sigma\right)$} & $r(\mathrm{CN})$ & 1.1813 & 1.1633 & 1.1605 & 1.1589 & & 1.1643 & & & & 1.1644 & 1.1572 & & & \multirow{2}{*}{ 36a, $r_{e}$} \\
\hline & $r(\mathrm{CH})$ & 1.0781 & 1.0593 & 1.0619 & 1.0636 & 1.0816 & 1.0616 & 1.0647 & 1.0666 & 1.0823 & 1.0669 & 1.0654 & 1.0673 & 1.0659 & \\
\hline \multirow[t]{2}{*}{$\mathrm{HNC}\left({ }^{1} \Sigma\right)$} & $r(\mathrm{HN})$ & 1.0055 & 0.9930 & 0.9939 & 0.9945 & 1.0064 & 0.9936 & 0.9946 & 0.9952 & 1.0067 & 0.9964 & 0.9949 & 0.9955 & 0.9941 & \multirow{2}{*}{$36 \mathrm{~b}, r_{e}$} \\
\hline & $r(\mathrm{NC})$ & 1.1912 & 1.1719 & 1.1703 & 1.1695 & 1.1975 & 1.1775 & 1.1758 & 1.1749 & 1.1973 & 1.1810 & 1.1756 & 1.1747 & 1.1689 & \\
\hline $\mathrm{CO}\left({ }^{1} \Sigma^{+}\right)$ & $r(\mathrm{CO})$ & 1.1467 & 1.1353 & 1.1321 & 1.1302 & 1.1545 & 1.1425 & 1.1390 & 1.1370 & 1.1552 & 1.1502 & 1.1397 & 1.1377 & 1.1283 & $31, r_{e}$ \\
\hline \multirow[t]{2}{*}{$\mathrm{NH}_{3}\left({ }^{1} A_{1}\right)$} & $r(\mathrm{NH})$ & 1.0226 & 1.0088 & 1.0077 & 1.0071 & 1.0258 & 1.0115 & 1.0106 & 1.0101 & 1.0267 & 1.0146 & 1.0115 & 1.0110 & 1.0124 & \multirow{2}{*}{$34 \mathrm{a}, r_{e}$} \\
\hline & $\theta(\mathrm{HNH})$ & 103.96 & 106.34 & 106.63 & 106.79 & 103.61 & 106.02 & 106.30 & 106.46 & 103.55 & 105.65 & 106.24 & 106.40 & 106.67 & \\
\hline $\mathrm{N}_{2}\left({ }^{1} \sum_{g}^{+}\right)$ & $r(\mathrm{NN})$ & 1.1294 & 1.1103 & 1.1078 & 1.1065 & 1.1266 & 1.1099 & 1.1079 & 1.1068 & 1.1216 & 1.1089 & 1.1029 & 1.1018 & 1.0977 & $31, r_{e}$ \\
\hline \multirow[t]{3}{*}{$\mathrm{N}_{2} \mathrm{H}_{2}\left({ }^{1} A_{g}\right)$} & $r(\mathrm{NH})$ & 1.0402 & 1.0257 & 1.0248 & 1.0243 & 1.0436 & 1.0285 & 1.0279 & 1.0276 & 1.0430 & 1.0279 & 1.0273 & 1.0270 & 1.0284 & \multirow{3}{*}{$34 \mathrm{~b}, r_{0}$} \\
\hline & $r(\mathrm{NN})$ & & 1.2509 & & 1.2474 & & & & & & & & 487 & 1.2517 & \\
\hline & $\theta(\mathrm{NN}$ & 104 & 105.63 & 105.91 & 106.07 & .78 & 105.61 & 105. & 106.04 & & 105.78 & 106.05 & 6.21 & 106.85 & \\
\hline \multirow[t]{3}{*}{$\mathrm{HNO}\left({ }^{1} A^{\prime}\right)$} & $r(\mathrm{NO})$ & 1.2257 & 1.2168 & 1.2147 & 1.2135 & 1.2260 & 1.2202 & 1.2188 & 1.2180 & 1.2233 & 1.2175 & 1.2161 & 1.2153 & 1.21 & \multirow{3}{*}{$38, r_{0}$} \\
\hline & $r(\mathrm{HN})$ & & & & & & & & & & & & & & \\
\hline & $\theta(\mathrm{HNO})$ & 107.37 & 107.67 & 107.76 & 107.81 & 107.55 & 107.77 & 107.82 & 107.84 & 107.68 & 107.89 & 107.94 & 107.97 & 108.58 & \\
\hline \multirow[t]{2}{*}{$\mathrm{H}_{2} \mathrm{O}\left({ }^{1} A_{1}\right)$} & $r(\mathrm{OH})$ & 0.9643 & 0.9576 & 0.9561 & 0.9552 & 0.9654 & 0.9585 & 0.9572 & 0.9565 & 0.9656 & 0.9599 & 0.9574 & 0.9567 & 0.9572 & \multirow{2}{*}{$35 \mathrm{a}, r_{e}$} \\
\hline & $\theta(\mathrm{HOH})$ & 101.96 & 103.65 & 104.15 & 104.45 & 101.90 & 103.60 & 104.11 & 104.40 & 101.86 & 103.50 & 104.07 & 104.36 & 104.52 & \\
\hline \multirow[t]{4}{*}{$\mathrm{H}_{2} \mathrm{O}_{2}\left({ }^{1} A\right)$} & $r(\mathrm{OO})$ & 1.4560 & 1.4466 & 1.4433 & 1.4415 & 1.4692 & 1.4587 & 1.4564 & 1.4551 & 1.4706 & 1.4601 & 1.4578 & 1.4565 & 1.4556 & \multirow{4}{*}{$35 \mathrm{~b}, r_{e}$} \\
\hline & $r(\mathrm{OH})$ & & & & 0.9 & & & & & & 540 & & 521 & & \\
\hline & $\theta(\mathrm{OOH})$ & & & & 99.94 & & & & & & & & & & \\
\hline & $\tau(\mathrm{HOOH})$ & 118.86 & 113.91 & 112.67 & 111.95 & 118.09 & 114.13 & 112.89 & 112.18 & 117.94 & 113.97 & 112.74 & 112.02 & 113.70 & \\
\hline $\mathrm{FH}\left({ }^{1} \Sigma^{+}\right)$ & $r(\mathrm{HF})$ & 0.9192 & 0.9170 & 0.9161 & 0.9157 & 0.9194 & 0.9171 & 0.9165 & 0.9162 & 0.9199 & 0.9178 & 0.9173 & 0.9170 & 0.9169 & $31, r_{e}$ \\
\hline $\mathrm{F}_{2}\left({ }^{1} \sum_{g}^{+}\right)$ & $r(\mathrm{FF})$ & 1.4239 & 1.3958 & 1.3953 & 1.3951 & 1.4504 & 1.4151 & 1.4149 & 1.4149 & 1.4596 & 1.4187 & 1.4185 & 1.4185 & 1.4119 & $31, r_{e}$ \\
\hline $\operatorname{HOF}\left({ }^{1} A^{\prime}\right)$ & $r(\mathrm{OH})$ & 0.9732 & 0.9658 & 0.9646 & 0.9639 & 0.9746 & 0.9673 & 0.9664 & 0.9659 & 0.9743 & 0.9670 & 0.9661 & 0.9656 & 0.9657 & $39, r_{e}$ \\
\hline & $r(\mathrm{OF})$ & & & & & & & & & & & & & & \\
\hline & $\theta(\mathrm{HOF})$ & 97.19 & 97.80 & 98.00 & 98.11 & 96.55 & 97.20 & 97.35 & 97.43 & 96.41 & 97.06 & 97.21 & 97.29 & 97.54 & \\
\hline & & & & & & & & & & & & & & & \\
\hline bond & $|\mu|$ & 0.0119 & 0.0061 & 0.0060 & 0.0060 & & & & & & 0.0053 & 0.0034 & 0.0033 & & \\
\hline lengths & $\mu$ & & -0.0022 & -0.0034 & -0.0040 & & & & & & & & 0.0014 & & \\
\hline & $\sigma$ & 0.0083 & 0.0077 & 0.0071 & 0.0069 & 0.0091 & 0.0059 & 0.0051 & 0.0047 & 0.0101 & 0.0062 & 0.0044 & 0.0043 & & \\
\hline bond & $|\mu|$ & 2.09 & 1.03 & 0.86 & 0.80 & 2.19 & 1.04 & 0.84 & & & & 0.80 & 0.68 & & \\
\hline & $\mu$ & & & & & & & & & & & -0.80 & -0.68 & & \\
\hline & $\sigma$ & 0.96 & 0.90 & 0.88 & 0.89 & 0.92 & 0.89 & 0.82 & 0.80 & 0.93 & 0.82 & 0.80 & 0.77 & & \\
\hline
\end{tabular}

${ }^{a}$ Bond lengths are given in $\AA$, angles in degrees. Values in italics denote geometrical parameters obtained by extrapolation. $\mathrm{CBS}=\mathrm{cc}-\mathrm{pV} \infty \mathrm{Z}$ limit values were determined with eq $1 .|\mu|, \mu$, and $\sigma$ denote mean absolute, mean, and standard deviation calculated according to eqs $2-4$.

with the mean absolute deviation $|\mu|$ in Table 3 separately for bond lengths and bond angles.

A number of conclusions can be drawn from the error analysis presented in Table 3 and Figures 2 and 3:

(1) With increasing basis set size, calculated geometries are improved both with regard to bond lengths and bond angles. However, these trends are somewhat more pronounced for MP6 geometries than MP4 geometries while MP2 geometries hardly change for cc-pVmZ basis sets with $m>3$.

(2) Also, with increasing basis set size bond lengths become shorter. These trends are well-known for MP2 and MP4, but they are confirmed in this work for MP6 geometries. At the same time the scattering of errors decreases as is reflected by the increasing maxima and the smaller standard deviations (halfwidth of normal distributions, Figures 2 and 3).

(3) For the cc-pVDZ basis set, bond lengths are always too long. With increasing order $n$ (MP2, MP4, MP6) the deviation from experimentally determined bond lengths increase rather than decrease as one might expect for a stepwise improved description of the molecule.

(4) For the cc-pVTZ basis set, calculated MP2 bond lengths are already too short on the average compared to the experimental values while the corresponding MP4/cc-pVTZ bond lengths are slightly longer than experimental values. However,
MP6/cc-pVTZ bond lengths are somewhat less accurate than MP4 values.

(5) With the cc-pVQZ basis set set, both MP4 and MP6 perform equally well with a slightly better agreement between experimental and MP6 bond lengths.

(6) At the CBS limit, MP6 leads to the best set of bond lengths for the three methods compared although the differences between MP6 and MP4 results are relatively small.

(7) Trends in calculated bond angles are directly related to trends in calculated bond lengths in the way that decreasing bond lengths imply increasing bond angles and vice versa.

These trends can be easily rationalized on the following basis. At the Hartree-Fock (HF) level of theory, an increase of the basis set size leads to an improved description of the valence shell of an atom thus making it possible that electron density is packed more closely around a nucleus in a molecule. With the larger basis set, the nuclei are better screened and as a consequence nuclear repulsion is reduced thus leading to a smaller bond length. In a three-atom situation, smaller bond lengths imply stronger 1,3 electrostatic repulsions and a widening of the bond angle, which explains the reverse relationship between trends in bond lengths and trends in bond angles.

Although this model description considers just the most relevant energy changes (Coulomb attraction between nuclei 


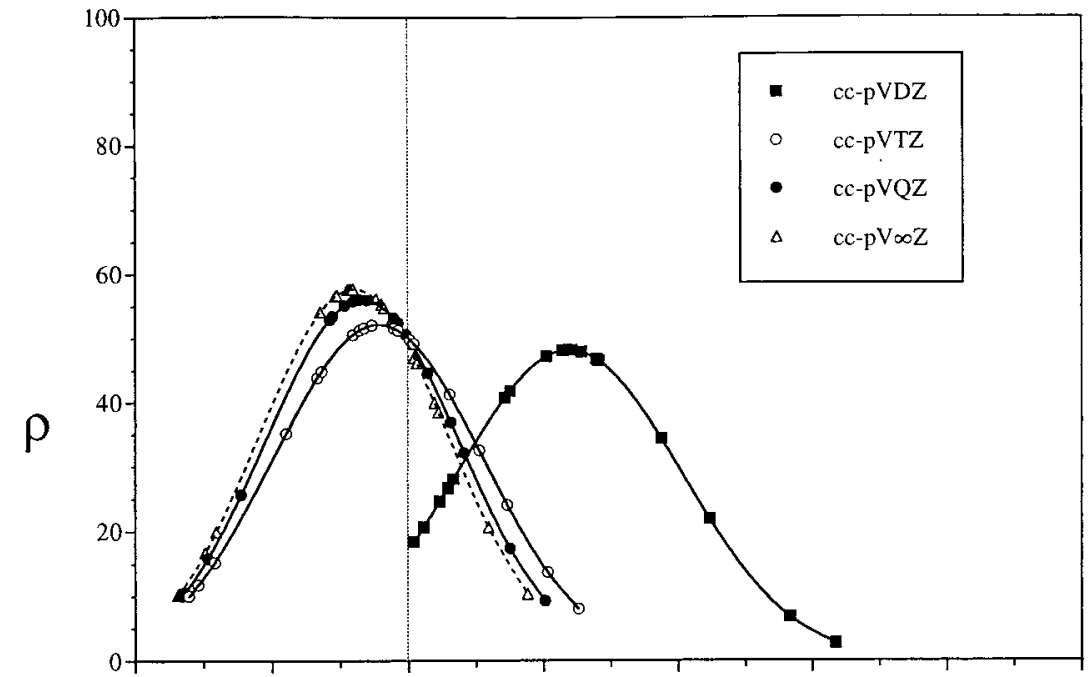

(a)

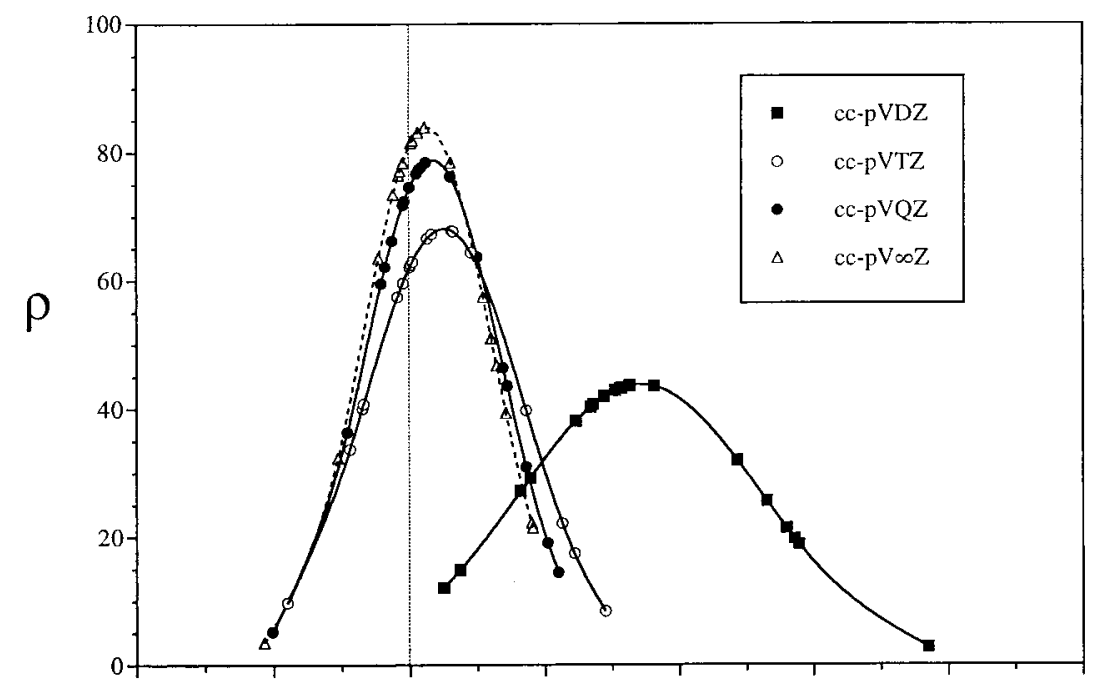

(b)

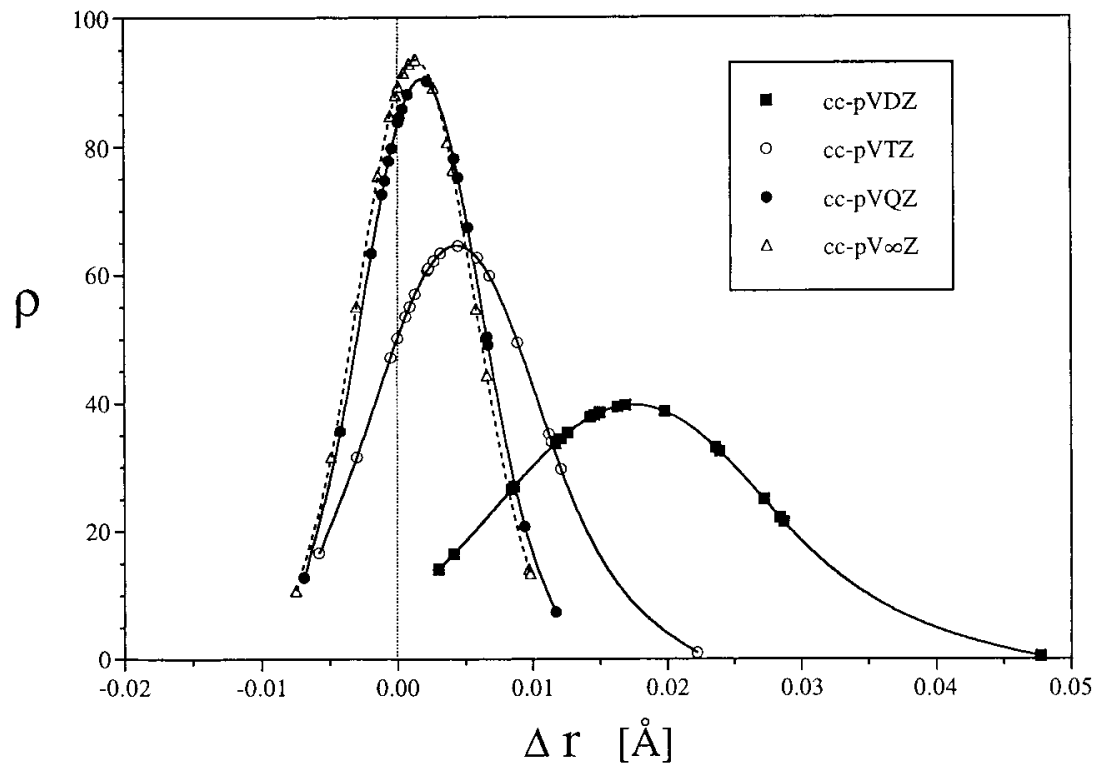

(c)

Figure 2. Normal distribution of errors for calculated MPn geometries using (a) the MP2/cc-pVmZ, (b) the MP4/cc-pVmZ, and (c) the MP6/ccpVmZ level of theory with $m=2,3,4$, and $\infty$ (CBS limit).

and electrons) and disregards other important effects (e.g., distribution of bonding electrons and electron lone pairs depending on the basis set used), it predicts typical trends in HF geometries correctly. MP2 introduces pair correlation effects in form of left-right, angular, and in-out correlation. ${ }^{2}$ The bonding electrons are separated in the bond region resulting in increased shielding (due to left-right correlation), but predominantly deshielding of the nuclei because angular and in-out correlation effects shift electron density away from the nuclei. Hence, the MP2 bond lengths are significantly longer than HF bond lengths calculated with the same basis set, where a relatively rigid basis set such as cc-pVDZ exaggerates these 

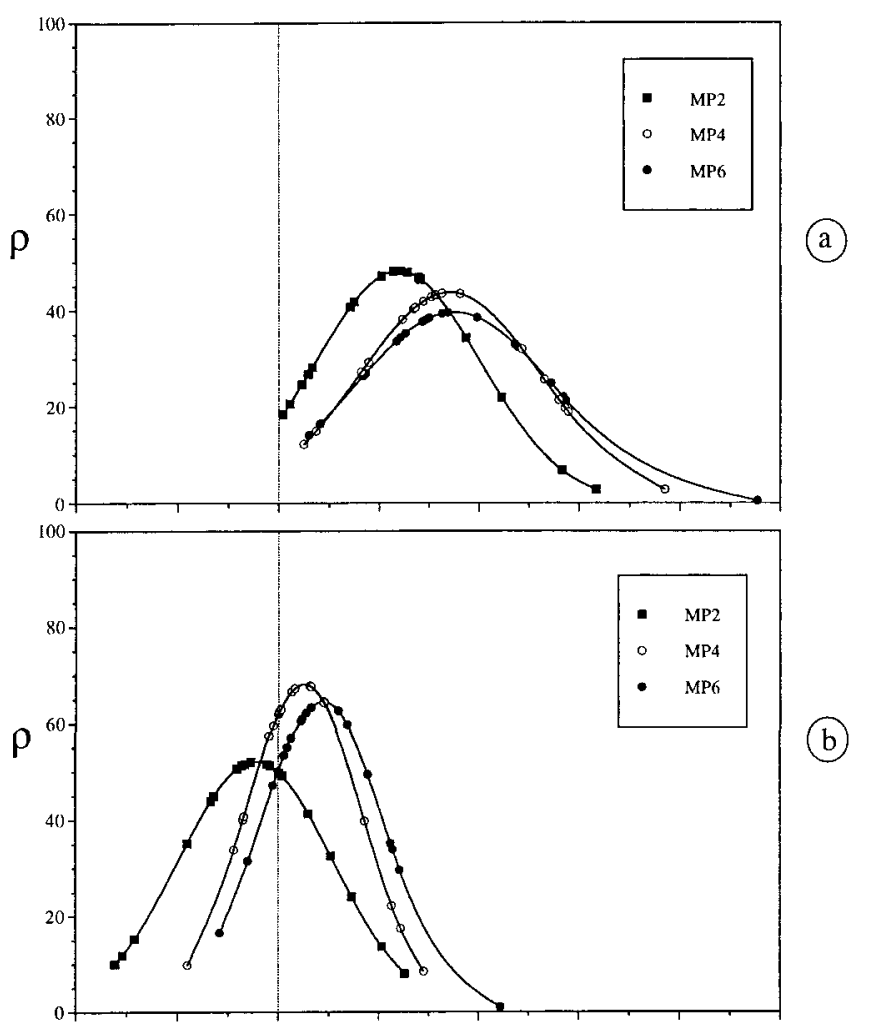
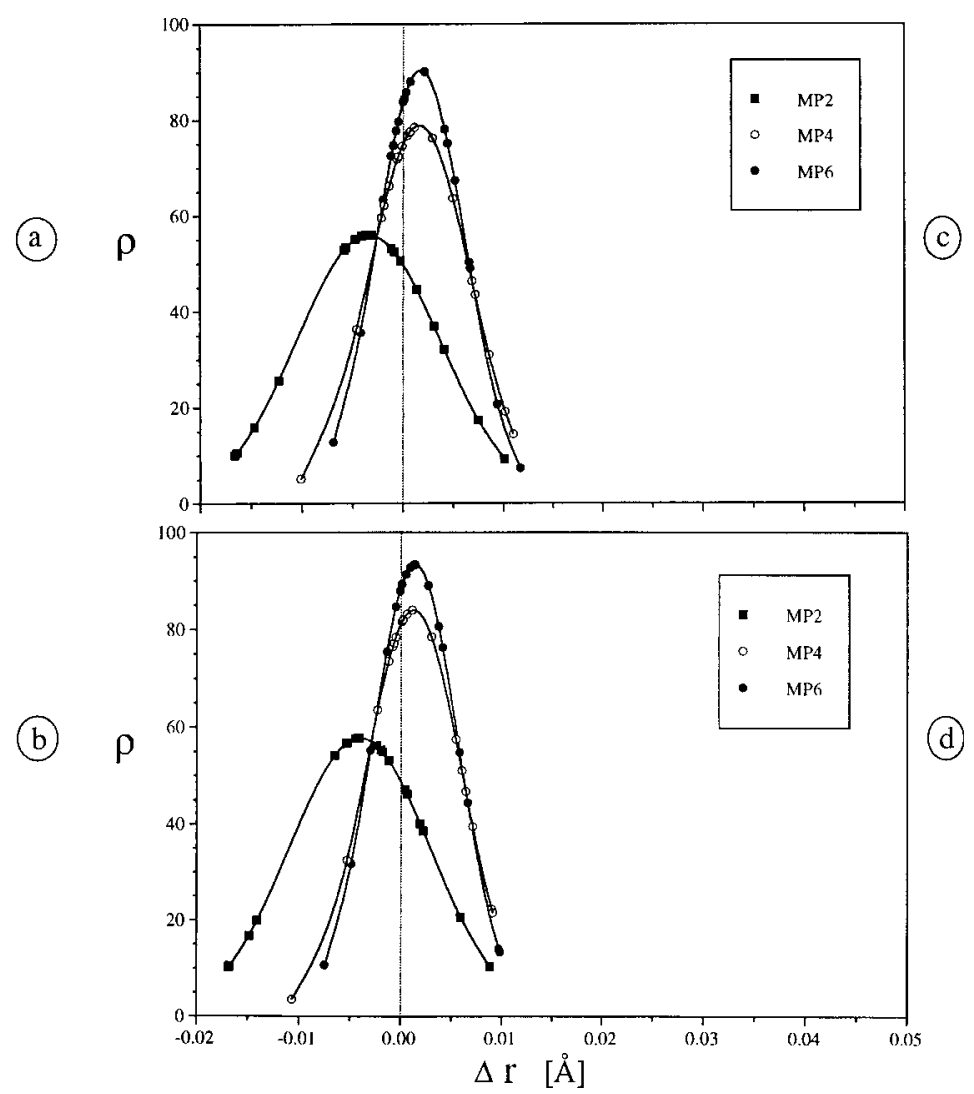

Figure 3. Normal distribution of errors for calculated MPn geometries obtained with (a) the cc-pVDZ, (b) the cc-pVTZ, (c) the cc-pVQZ, and (d) the CBS limit basis at MP $n$ orders $n=2,4$, and 6 .

effects. The more basis functions included in the basis the more it will be possible to separate electron pairs but at the same time to pack electrons into the inner valence sphere thus increasing stabilizing nucleus-electron attractions.

At MP4, three-electron correlation effects make it possible that of three electrons in the region of a multiple bond at least one is shifted in a more angular or outer position while the two other are left-right correlated. This implies directly that at MP4 because of three-electron correlation the nuclei are more deshielded than at MP2 and, accordingly, bond lengths become longer. Again, an increasingly flexible basis set leads to better compromises between electron separation and packing of electrons around the nuclei which explains the decrease in bond lengths with increasing basis set size.

The improvements in electron correlation, which enter at MP6, concern in particular four-electron and six-electron situations. Connected Q excitations make it possible that the four electrons of a double bond are left-right and top-bottom (angular) correlated thus leading to an even stronger deshielding of the nuclei and longer bond lengths. Disconnected six-electron correlation effects are important for structures with triple bonds (or single bonds involving heteroatoms with electron lone pairs). Although the three electron pairs described by an $\mathrm{H}$ excitation at MP6 are disconnected they are coupled to disconnected Q correlations, which leads to a coupling correction in the way that the pair correlation effects are not independent. Hence, typical left-right, top-bottom patterns result from these correlation effects, again leading to an overall deshielding of the nuclei, which is most drastic for the relatively rigid $\mathrm{VDZ}+\mathrm{P}$ basis set. Flexibility in the basis set is now a must to balance effects of electron separation on the one side and density contraction toward the nuclei on the other side.
The more rigid a basis set is the less it is capable of correctly describing higher order electron correlation effects. Hence, if a $\mathrm{VDZ}+\mathrm{P}$ basis set is used in connection with a method that includes connected three- and four-electron correlation effects, these higher electron correlation effects are artificially suppressed and replaced by exaggerated pair correlation effects. Hence, geometries calculated with a VDZ+P basis set deviate stronger from experimental values the higher the level of MPn theory is applied. If one improves the method to cover more electron correlation, one has to invest at the same time into the basis set despite the fact that the typical $\mathrm{O}\left(\mathrm{M}^{n+3}\right)$ dependence increases calculational cost enormously. We conclude that reliable MP6 geometries are hardly feasible unless suitable extrapolation methods are used.

\section{Quality of MP6 Complete Basis Set Geometries}

In Figure 3, trends in calculated CBS bond lengths are given in dependence of the order $n$ of MP theory applied and compared with the corresponding experimental values. A comparison with CBS limit values obtained with other methods ${ }^{26,27,29}$ is given in Table 4.

As Figure 4 reveals and Table 3 gives in detail, MP6/CBS limit values (similar to MP6/cc-pVTZ values) provide an accurate account of $\mathrm{AH}$ single bond lengths. The only exception is found for HNO, however in this case the experimental $r_{0}$ value $^{38}$ may differ from the correct $r_{e} \mathrm{NH}$ bond length. In any case, the MP6 AH bond lengths are more accurate than MP2/ CBS, MP4/CBS, and CCSD(T)/CBS values reported in the literature (Table 4).

Reasonable values are also obtained for the $\mathrm{OO}$ bond in $\mathrm{H}_{2} \mathrm{O}_{2}$, the $\mathrm{NN}$ bonds in $\mathrm{N}_{2} \mathrm{H}_{2}$ and $\mathrm{N}_{2}$ as well as for the $\mathrm{NC}$ bond in HCN. However, deviations between experimental and MP6/CBS 
TABLE 4: Comparison of CBS Limit Geometries Taken from the Literature or Calculated in This Work with the Corresponding Experimental Values ${ }^{a}$

\begin{tabular}{|c|c|c|c|c|c|c|c|c|}
\hline \multirow[b]{2}{*}{ method } & \multirow{2}{*}{$\begin{array}{c}\mathrm{FH} \\
r(\mathrm{FH})\end{array}$} & \multicolumn{2}{|c|}{$\mathrm{CH}_{2}\left({ }^{3} B_{1}\right)$} & \multirow{2}{*}{$\begin{array}{c}\mathrm{CH}_{4} \\
r(\mathrm{CH})\end{array}$} & \multirow{2}{*}{$\begin{array}{c}\mathrm{CO} \\
r(\mathrm{CO})\end{array}$} & \multirow{2}{*}{$\begin{array}{c}\mathrm{F}_{2} \\
r(\mathrm{FF})\end{array}$} & \multirow{2}{*}{$\begin{array}{c}\mathrm{N}_{2} \\
r(\mathrm{NN})\end{array}$} & \multirow[b]{2}{*}{ ref } \\
\hline & & $r(\mathrm{CH})$ & $\theta(\mathrm{HCH})$ & & & & & \\
\hline $\mathrm{SCF}$ & & -5.9 & -4.45 & -4.2 & -26.2 & -85.6 & & Lit. \\
\hline \multirow[t]{2}{*}{ MP2 } & 0.8 & -3.0 & -0.82 & -1.4 & 5.9 & -17.0 & 11.7 & Lit. \\
\hline & -1.3 & -5.4 & -0.89 & -4.5 & 2.2 & -16.8 & 8.8 & t.w. \\
\hline MP3 & -5.4 & -1.3 & -0.35 & -0.8 & -11.1 & -32.2 & -12.3 & Lit. \\
\hline \multirow[t]{2}{*}{ MP4 } & 1.4 & 0.9 & -0.25 & 2.2 & 12.8 & 2.4 & 11.4 & Lit. \\
\hline & -0.7 & -1.2 & -0.55 & -0.7 & 9.0 & 3.0 & 9.1 & t.w. \\
\hline MP6 & 0.1 & 0.5 & -0.34 & -0.1 & 9.7 & 6.6 & 4.1 & Lit. \\
\hline CCSD & -3.0 & 0.5 & -0.54 & 0.9 & -4.7 & & -5.7 & Lit. \\
\hline $\operatorname{CCSD}(\mathrm{T})$ & -0.2 & 1.9 & -0.36 & 2.3 & 2.5 & & 1.5 & Lit. \\
\hline \multirow[t]{2}{*}{ Exp. } & 0.9169 & 1.0753 & 133.93 & 1.0857 & 1.1283 & 1.4119 & 1.097 & \\
\hline & 31 & $33 a$ & $33 a$ & $33 b$ & 31 & 31 & 31 & Lit. \\
\hline
\end{tabular}

${ }^{a}$ Experimental bond lengths and bond angles are given in $\AA$ and degrees, deviations of calculated bond lengths and bond angles from the corresponding experimental values in $10^{-3} \AA$ and degrees. Literature (Lit.) values from refs $26 \mathrm{e}\left(\mathrm{FH}, \mathrm{F}_{2}, \mathrm{~N}_{2}\right), 27(\mathrm{CO})$, and $29\left(\mathrm{CH}_{2}, \mathrm{CH}_{4}\right)$. Values for $\mathrm{CH}_{2}\left({ }^{3} B_{1}\right)$ and $\mathrm{CH}_{4}$ correspond to cc-pV5Z/QZ calculations where $\mathrm{CH}_{2}\left({ }^{3} B_{1}\right)$ was calculated using restricted open shell theory. The abbreviation t.w. indicates results obtained in this work.

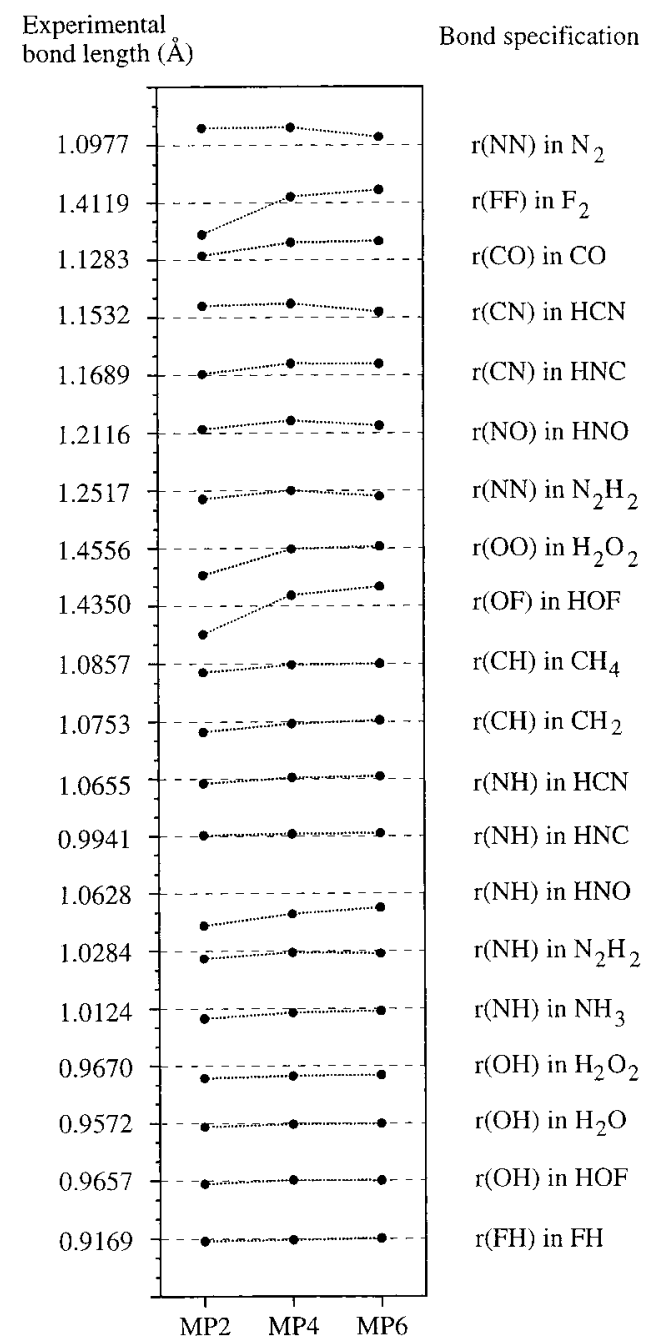

Figure 4. Graphical presentation of CBS bond lengths in dependence of the MP $n$ order $n$.

heavy atom bond lengths are relatively large for $\mathrm{F}_{2}, \mathrm{CO}, \mathrm{HNC}$, and HOF predicting these bond lengths too long at all levels of MP6 theory. Since the CBS limit should not suffer from any remaining basis set deficiencies (although the extrapolation procedure could lead to some inaccuracies as discussed in the previous section), deviations of 0.004 up to $0.010 \AA$ indicate correlation errors, which seem not to be present at the CCSD(T) level of theory (see Table 4).
MP6 can correlate at most three electron pairs at the same time as was discussed in the previous chapter. If there are four or even five electron pairs close to the bond region $(\mathrm{O}-\mathrm{F}$ : 1 bonding electron pair $+1 \sigma$-type electron lone pair $+3 \pi$-type electron lone pairs; F-F: $1+4$; etc.) correlation at MP6 does not include all electron lone pairs simultaneously. Hence, one has to discuss what would happen if four or five electron pairs would be correlated at higher levels of MP $n$ theory (MP8 or MP10). Clearly, electrons would be rearranged so that (a) they avoid each other as much as possible, (b) they can approach the nuclei as much as possible, and (c) they stay in the bonding region as much as possible. This would imply that lone pair electrons become somewhat more diffuse allowing the bonding electron pairs to stay in the bond region but at the same time to move closer to the nuclei. If electron lone pairs move further away from a nucleus, this corresponds to an increase of effective electronegativity of the atom in question with regard to the bonding electrons. The nucleus is stronger shielded by the bonding electrons and the bond length becomes shorter, which is required in the case of the $\mathrm{O}-\mathrm{F}, \mathrm{F}-\mathrm{F}$, and $\mathrm{CO}$ bond to obtain a better agreement with experiment. Therefore, the observed deviations of the MP6/CBS values reflect the shortcomings of the MP6 approach.

In the case of $\mathrm{N}_{2}$, the electron lone pairs are symmetrically arranged at the backside of the bond region and, therefore, the error in the MP6/CBS bond length is smaller than for the $\mathrm{CO}$ molecule (Figure 4). Although the electron lone pairs are also relatively far away from the bond region of the $\mathrm{CO}$, their influence on the bonding situation is much larger than in the case of $\mathrm{N}_{2}$ since they indirectly determine the polarity of the $\mathrm{CO}$ bond and by this the screening of the $\mathrm{C}$ and $\mathrm{O}$ nuclei in the bond region. Similar considerations apply to the OF bond in $\mathrm{HOF}$, the $\mathrm{NC}$ bond in $\mathrm{HNC}$, and the $\mathrm{NO}$ bond in $\mathrm{HNO}$ (Figure 4).

The electron correlation effects just described would be automatically covered by a correlation corrected ab initio method with infinite order pair and three-electron correlation effects as done by $\operatorname{CCSD}(\mathrm{T})$. CCSD covers just the pair correlation effects and as reflected by the CCSD/CBS values for $\mathrm{F}_{2}, \mathrm{~N}_{2}$, and $\mathrm{CO}$ (Table 4) is already better than MP6 but not as accurate as $\operatorname{CCSD}(\mathrm{T})$ (Table 4).

\section{Conclusions}

A new method to predict CBS limit geometries at higher orders of MP $n$ theory from CBS values at lower orders of $\mathrm{MP} n$ theory is used to determine MP6/CBS geometries. This 
method is related to the procedures described by Pople and coworkers for obtaining reliable heats of formations, dissociation enthalpies, ionization potentials, and electron affinities. ${ }^{20}$ From the investigation of 14 small molecules, for which reliable experimentally based $r_{e}$ geometries are known, conclusions (1)-(6) can be drawn.

(1) Trends in calculated MP2, MP4, and MP6 geometries with regard to the basis set employed are parallel. For example, calculated bond lengths in general decrease (bond angles widen) when the basis set is increased to the CBS limit. These changes can be explained when considering the redistribution of electrons made possible by a better description of molecular space with the help of larger basis sets.

(2) For MP2, a fortuitous cancellation of basis set and correlation errors in the case of basis sets with VTZ $+\mathrm{P}$ quality leads to surprisingly reliable equilibrium geometries. For MP4 and MP6, calculated bond lengths are on the average always longer than experimental values and at least a VTZ $+\mathrm{P}$ basis set is required to get reasonable geometries.

(3) The best compromise between accuracy and economy of an MP calculation is obtained with a cc-pVTZ basis either at the MP2 or (somewhat more reliable) at the MP4 level of theory. For saturated molecules without any heteroatoms the use of a cc-pVQZ basis at MP2 seems to be meaningless although it will be necessary for molecules with electron clustering. At $\mathrm{MP} 4$, it is more important to use the VQZ+P basis set.

(4) Accurate MP6 geometries are obtained for molecules without heteroatoms or for $\mathrm{H}_{n} \mathrm{AAH}_{n}$ molecules with a symmetrical arrangement of the heteroatoms provided a cc-pVTZ or larger basis set is employed. MP6 fails however for principal reasons when describing polar bonds involving one or two heteroatoms. Infinite order pair and three-electron correlation effects as covered by $\operatorname{CCSD}(\mathrm{T})$ are needed for the calculation of accurate geometries.

(5) Relative to MP4, MP6 leads to slightly improved geometries.

(6) MP6/CBS values reveal that the MPn series converges to the true equilibrium geometry of electron systems with electron clustering (single and multiple bonds between electronegative heteroatoms) rather slowly and, therefore, cannot be considered to be the right method for high-accuracy calculations. The basic deficiency of MP methods is the lack of infinite order effects contained in CC methods. This explains why CCSD(T)/CBS geometries and sometimes even CCSD/CBS geometries are superior to MP6/CBS geometries.

(7) As for the question posed in the title of this article, the present work provides an answer for the geometries of molecules made up from first row atoms $(\mathrm{Li}-\mathrm{Ne})$. Molecules with little electron clustering will already be reasonably described at MP4. However, with an increase of electron clustering as found in the vicinity of electronegative atoms, in the region of multiple bonds, etc., MP6 and even higher MP $n$ methods have to be applied to obtain accurate geometries. In the worst case, a bond length may be influenced by the interaction of up to five electron pairs (e.g., F-F: 1 bonding electron pair $+4 \mathrm{p} \pi$-type electron lone pairs; the two p $\sigma$-type electron pairs influence the $\mathrm{F}-\mathrm{F}$ bond length only indirectly). Such a bond length can correctly be predicted if the simultaneous correlation of five electron pairs is covered by the MPn method in question. Hence MP10, which includes for the first time disconnected 10-fold excitations (independent but simultaneous correlation of five electron pairs), is necessary to guarantee an exact description of FF, OF, or $\mathrm{OO}$ bonds. Considering the large number of terms covered by MP8 (915) or MP10 (16 605) as well as their high cost $\left(\mathrm{O}\left(\mathrm{M}^{11}\right)\right.$ and $\mathrm{O}\left(\mathrm{M}^{13}\right)$, there is little chance that these methods will ever be developed and programmed for routine use.

One cannot expect reliable geometries from MP6/cc-pVDZ calculations or other VDZ+P basis sets because pair correlation effects are artificially exaggerated leading to too long bonds and too short angles. A VTZ $+\mathrm{P}$ or even $\mathrm{VQZ}+\mathrm{P}$ basis set is necessary to get reasonable MP6 geometries. We confirm for geometries with what is generally known, namely that with increased coverage of electron correlation effects the requirements for the basis set (saturation of the sp-basis, increased flexibility by even-tempered polarization functions covering all regions of molecular space in a balanced way) increase. Improvements of method and basis set have to be parallel. This clearly limits the use of MP6 in quantum chemistry. Therefore one can conclude that for the case that MP4 fails to provide a satisfactory geometry, it is advisable in most cases to apply $\operatorname{CCSD}(\mathrm{T})$ rather than higher orders of MPn theory. Exceptions such as FOOF (MP6 provides a considerably more accurate geometry than $\mathrm{CCSD}(\mathrm{T}))$ recently investigated by Kraka and co-workers ${ }^{41}$ do not contradict this rule of thumb.

Acknowledgment. This work was supported by the Swedish Natural Science Research Council (NFR). All calculations were done on the CRAY C90 of the Nationellt Superdatorcentrum (NSC), Linköping, Sweden. The authors thank the NSC for a generous allotment of computer time.

\section{References and Notes}

(1) Hehre, W. J.; Radom, L.; Schleyer, P. v. R.; Pople, J. A. Ab Initio Molecular Orbital Theory; Wiley: New York, 1986.

(2) For a recent review see, Cremer, D. In Encyclopedia of Computational Chemistry; Schleyer, P. v. R. Allinger, N. L., Clark, T., Gasteiger, J., Kollman, P. A., Schaefer III, H. F., Schreiner, P. R., Eds.; John Wiley: Chichester, U.K., 1998; Vol. 3, p 1706.

(3) Gauss, J.; Cremer, D. Adv. Quantum Chem. 1992, 23, 205.

(4) DeFrees, P. J.; Levi, B. A.; Pollack, S. K.; Hehre, W. J.; Binkley, J. S.; Pople, J. A. J. Am. Chem. Soc. 1991, 113, 1507. 7846.

(5) Johnson, B. G.; Gill, P. M.; Pople, J. A. J. Chem. Phys. 1992, 97,

(6) Scuseria, G.; Lee, T. L. In Quantum Mechanical Electronic Structure Calculations with Chemical Accurary; Langhoff, S. R., Ed.; Kluwer Academics: Dordrecht, 1995; p 47.

(7) Schaefer, H. F., III; Thomas, J. R.; Yamaguchi, Y.; Deleeuw, B. J.; Vacek, G. In Modern Electronic Structure Theory, Yarkony, D. R., Ed.; World Scientific: Singapore, 1995.

(8) Oliphant, N.; Bartlett, R. J. J. Chem. Phys. 1994, 100, 6550.

(9) Helgaker, T.; Gauss, J.; Jørgenson, P.; Olsson, J. J. Chem. Phys. 1997, 106, 6430.

(10) Asger, H.; Jørgenson, P.; Gauss, J.; Helgaker, T. Chem. Phys. Lett. 1997, 274, 235. 7190 .

(12) Gauss, J.; Cremer, D.; Stanton, J. F. J. Phys. Chem. 2000, 104, 1319.

(13) (a) Harmony M. D. Acc. Chem. Res. 1992, 25, 321. (b) Kuchitsu, K.; Cyvin, S. J. In Molecular Structure and Vibrations; Cyvin, S. J., Ed.; Elsevier: Amsterdam, 1972; pp 183-211. (c) Accurate Molecular Structures, Domenicano, A., Hargittai, I., Ed.; Oxford University Press: Oxford, U.K., 1992; p 355.

(14) Møller, C.; Plesset, M. S. Phys. Rev. 1934, 46, 618.

(15) He, Z.; Cremer, D. Int. J. Quantum Chem. 1996, 59, 15; 31; 57;

(16) Cremer, D.; He, Z. J. Phys. Chem. 1996, 100, 6173.

(17) Cremer, D.; He, Z. J. Mol. Struct. (THEOCHEM) 1997, 398-399,

(18) (a) Peterson K. A.; Woon, D. E.; Dunning, T. H., Jr. J. Chem. Phys. 1994, 100, 7410. (b) van Mourik, T.; Wilson, A. K.; Dunning, T. H., Jr. Mol. Phys. 1999, 96, 529.

(19) (a) Martin, J. R. L.; Taylor, P. R. Chem. Phys. Lett. 1994, 225 , 473. (b) Martin, J. M. L.; Lee, T. J. Chem. Phys. Lett. 1996, 258, 136. 
(20) (a) Curtiss, L. A.; Ragavachari, K.; Trucks, G. W.; Pople, J. A. J. Chem. Phys. 1991, 94, 7221. See also (b) Curtiss, L. A.; Raghavachari, K.; Redfern, P. C.; Rassolov, V.; Pople, J. A. J. Chem. Phys. 1998, 109, 7764. (21) (a) Christiansen, O.; Olsen, J.; Jørgensen, P.; Koch, H.; Malmqvist, P. Chem. Phys. Lett. 1996, 261, 369. (b) Olsen, J.; Christiansen, O.; Koch, H.; Jørgensen, P. J. Chem. Phys. 1996, 105, 5082.

(22) (a) For a recent review see, Gauss, J. In Encyclopedia of Computational Chemistry, Schleyer, P. v. R., Allinger, N. L.; Clark, T., Gasteiger, J., Kollman, P. A., Schaefer, H. F., III, Schreiner, P. R., Eds.; John Wiley: Chichester, U.K., 1998; Vol. 1, p 615. (b) Bartlett, R. J.; Stanton, J. F. In Reviews in Computational Chemistry; Lipkowitz, K. B. Boyd, D. B., Eds.; VCH: Weinheim, Germany, 1994; Vol. 5, p 65. (c) Cremer, D.; He, Z. In Conceptual Perspectives in Ouantum Chemistry, Calais, J.-L., Kryachko, E., Eds.; Kluwer Publishing Co.: Dordrecht, The Netherlands, 1997; Vol. 3, p 239.

(23) Kraka, E.; Gräfenstein, J.; Gauss, J.; Reichel, F.; Olsson, L.; Konkoli, Z.; He, Z.; Cremer, D. COLOGNE 99, Göteborg University, Göteborg, 1999.

(24) (a) Fletcher, R.; Powell, M. J. D. Comput. J. 1963, 6, 163. (b) Davidon, W. Argonne National Lab. Report, ANL-5990.

(25) Dunning, T. H., Jr. J. Chem. Phys. 1989, 99, 1007.

(26) (a) Woon, D. E.; Dunning, T. H., Jr. J. Chem. Phys. 1993, 99, 1914. (b) Peterson, K. A.; Kendall, R. A.; Dunning, T. H., Jr. J. Chem. Phys. 1993, 99, 1930. (c) Peterson, K. A.; Kendall, R. A.; Dunning, T. H., Jr. J. Chem. Phys. 1993, 99, 9790. (d) Wilson, K. A.; Dunning, T. H., Jr. J. Chem. Phys. 1997, 106, 8718. (e) Wilson, A. K., van Mourik, T., Dunning, T. H., Jr. J. Mol. Struct. (THEOCHEM) 1996, 388, 339.

(27) Peterson, K. A.; Dunning, T. H., Jr. J. Mol. Struct. (THEOCHEM) 1997, 400, 93 4761.

(29) Peterson, K. A.; Dunning, T. H., Jr. J. Chem. Phys. 1997, 106, 4119.

(30) Forsberg, B.; He, Z.; He, Y.; Cremer, D. Int. J. Quantum Chem. 2000, 76, 306.

(31) Huber, K. P.; Herzberg, G. H. Molecular Spectra and Molecular Structure IV: Constants of Diatomic Molecules; Van Nostrand-Reinhold: New York, 1979.

(32) Chase, M. W., Jr.; Davles, C. A.; Downey, J. R., Jr.; Frurip, D. J.; McDonald, R. A.; Syverud, A. N. JANAF Thermochemical Tables, J. Phys. Chem. Ref. Data 14; 1985, Suppl., 1.

(33) (a) $\mathrm{CH}_{2}\left({ }^{3} \mathrm{~B}_{1}\right)$ : Jensen, P.; Bunker, P. R. J. Chem. Phys. 1988, 89 , 1327. (b) $\mathrm{CH}_{4}$ : Gray, D. L.; Robiette, A. G. Mol. Phys. 1979, 37, 1901.

(34) (a) $\mathrm{NH}_{3}$ : Benedict, W. S.; Plyler, E. K. Can. J. Phys. 1957, 35, 1235. (b) $\mathrm{N}_{2} \mathrm{H}_{2}$ : Carlotti, M.; Johns, J. W. C.; Trombetti, A. Can. J. Phys. 1974, 52, 340 .

(35) (a) $\mathrm{H}_{2} \mathrm{O}$ : Hoy, A. R.; Mills, I. M.; Strey, G. Mol. Phys. 1972, 24, 1265. (b) $\mathrm{H}_{2} \mathrm{O}_{2}$ : Pelz, G.; Yamada, K. M. T.; Winnerwisser, G. J. Mol. Spectrosc. 1993, 159, 597.

(36) (a) HCN: Winnewisser, G.; Maki, A. G.; Johnson, D. R. J. Mol. Spectrosc. 1971, 39, 149. (b) HNC: Creswell, R. A.; Robiette, A. G. Mol. Phys. 1978, 36, 869.

(37) $\mathrm{CO}_{2}$ : Graner, G.; Rosetti, C.; Baily, D. Mol. Phys. 1986, 58, 627.

(38) HNO: Dalby, F. W. Can. J. Phys. 1958, 36, 1336.

(39) HOF: Halonen, L.; Ha, T.-K. J. Chem. Phys. 1988, 89, 4885.

(40) $\mathrm{O}_{3:}$ Tanaka, T.; Morino, Y. J. Mol. Spectrosc. 1970, 33, 538.

(41) Kraka, E.; He, Y.; Cremer, D. J. Phys. Chem., submitted. 